\title{
Siendo líder con inteligencias tecnológicas
}

\section{Being a leader with technological intelligence}

SÁNCHEZ-RIVERA， Lilia†*， ESPERICUETA-MEDINA， Marta Nieves, MUÑOZ-LÓPEZ, Temístocles y DELGADO-ESTRADA, Sandra

Universidad Autónoma de Coahuila - Facultad de Ciencia, Educación y Humanidades

ID $1^{\text {er }}$ Autor: Lilia, Sánchez-Rivera / ORCID: 0000-0001-9468-2599, Researcher ID Tomson: T-1404-2018, CVU CONACYT ID: 613195

ID $1^{\text {er }}$ Coautor: Marta Nieves, Espericueta-Medina / ORC ID: 0000-0002-4924-4332, Researcher ID T-1500-2018, arXiv Author ID: Espericueta2018, CVU CONACYT ID: 372705

ID $2^{\text {do }}$ Coautor: Temístocles, Muñoz-Lopez / ORC ID: 0000-0003-4940-5730, Researcher ID Tomson: T-7834-2018, CVU CONACYT ID: 202437

ID $3^{\text {er }}$ Coautor: Sandra, Delgado-Estrada / ORC ID 0000-0002-7056-4843, CVU CONACYT ID 1095193

DOI: $10.35429 /$ JET.2020.12.4.13.20

Recibido: 28 de Agosto, 2020; Aceptado 29 de Diciembre, 2020

\section{Resumen}

La publicación expone aspectos importantes del uso de las tecnologías de información y comunicación relacionadas con las inteligencias múltiples y liderazgo organizacional. El objetivo de este artículo fue explorar las dimensiones TIC, inteligencias múltiples y liderazgo organizacional. La metodología que se utilizó fue cuantitativa, transversal descriptiva y correlacional; la recopilación de la información fue a través de un cuestionario que median las tres dimensiones mencionadas. La muestra se conformó por 315 estudiantes, el muestro utilizado fue no probabilístico, mediante la estrategia de muestreo por conveniencia. Las técnicas estadísticas que se utilizaron fueron la estadística descriptiva, correlacional y factorial. La contribución del estudio radica en la evidencia empírica estadística que permite afirmar que el uso de las TIC benefician a las inteligencias múltiples y al liderazgo organizacional; se denota una correlación positiva con variables del uso de las TIC en relación con las inteligencias múltiples lingüística-verbal, lógicomatemático, espacial, interpersonal y con el liderazgo organizacional, se puede decir que las TIC de manera integrada con las inteligencias múltiples y con el liderazgo organizacional propicia ser un líder con inteligencia tecnológica.

Inteligencias múltiples, TIC, Liderazgo

\begin{abstract}
The article exposes important aspects of the usage of technologies of information and communication related to multiple intelligences and organizational leadership.The objective of this article was to explore the TIC's dimensions, multiple intelligences, and organizational leadership. The methodology used quantitative, transversal descriptive and correlational. The compilation of the information was through a questionary that measured the three dimensions mentioned. The sample was confirmed by 315 students, the sampling used was no probabilistic, through the strategy of convenience sampling. The statistics techniques that were used were descriptive statistic, correlational and factorial. The contribution of the study lies in the statistic empiric evidence that allows to confirm that use of the TIC benefit to the multiple intelligences and to the organizational leadership. It is denoted that the positive correlation with variables of the use of the TIC in relation with the multiple intelligences linguistic-verbal, logical-mathematical, space, interpersonal and with the organizational leadership, it can be said that the TIC integrated with multiple intelligences and the use of organizational leadership propitiate to be a leader with technological intelligence.
\end{abstract}

Multiple intelligences, TIC, Leadership

Citación: SÁNCHEZ-RIVERA，Lilia，ESPERICUETA-MEDINA， Marta Nieves，MUÑOZ-LÓPEZ，Temístocles y DELGADO-ESTRADA, Sandra. Siendo líder con inteligencias tecnológicas. Revista Teoría Educativa. 2020. 4-12: 13-20

\footnotetext{
* Correspondencia del Autor (1sr14712@uadec.edu.mx)

$\dagger$ Investigador contribuyendo como primer autor.
} 


\section{Introducción}

La popularización de las TIC en el ámbito educativo comporta y comportará en los próximos años, una gran revolución que contribuirá a la innovación del sistema educativo e implicará retos de renovación y mejora de los procesos de enseñanza-aprendizaje (Albero, 2002). Con el ánimo de mejorar la calidad educativa del país, los últimos esfuerzos de la política pública ha sido la implantación de programas orientados a la puesta en marcha de una educación cada vez más globalizada, en la que los estudiantes utilicen las tecnologías de la información y la comunicación (TIC) para mejorar su rendimiento académico (Castells,2010). Según Ferro et al. (2009), una consecuencia de la implementación de las TIC en el aprendizaje es la eliminación de las barreras espacio-temporales a las que se ha visto condicionada la enseñanza presencial. Desde este punto de vista, se asume que el aprendizaje ya no se produce en un espacio netamente físico sino digital, el ciberespacio, en el cual se tienden a desarrollar interacciones comunicativas mediáticas.

Vasovic et al., 2014 citado por Sánchez et al., 2019 señaló que:

Los avances tecnológicos impulsados por los desarrollos en el área de la computación, están cambiando de forma vertiginosa el estilo de vida en la sociedad actual; por ende, la educación superior, es sin duda uno de los sectores que está realizando esfuerzos por innovar en sus procesos educativos para dar respuesta a estas demandas con la incorporación de las TIC en sus procesos de enseñanza, sin embargo, la amplitud de herramientas que se consideran como parte de esta categoría, requiere de algunas precisiones teóricas para establecer de forma concreta qué son y cómo pueden aplicarse. En términos generales, resulta complejo establecer una definición única sobre las TIC, entendiendo que poseen características y propósitos diversos; sin embargo, es posible hacer una aproximación conceptual desde un punto de vista educativo, afirmando que son un conjunto de herramientas y recursos que estimulan y contribuyen a la interacción dinámica entre individuos facilitando así el aprendizaje y la divulgación de los saberes.
Bonami et al., 2020 mencionan que "una idea sobre cómo definir la tecnología es el concepto de «technium»: un ecosistema autorreforzante de creación de artefactos, herramientas e ideas que abarcan las tecnologías. Según esta idea, la tecnología depende de innumerables avances anteriores". Por su parte, la inteligencia ha sido uno de los campos de interés que más se ha estudiado en la Psicología, la cual ha querido dar respuesta y explicar un constructo tan abstracto como éste, encaminándose en la búsqueda de unas herramientas e instrumentos que hicieran visibles los conceptos que acerca de ella se tienen. Un recorrido por la posición de distintos teóricos permite encontrar tendencias extremadamente biologicistas y genéticas y otras que se van al plano de las relaciones humanas y conductas que al final todas confluyen en un punto común que es el desarrollo cognitivo y humano. Algunas concepciones de inteligencia que han creado diferentes estudiosos del área: (Sandoval, A., González, L., González, O., \& Lauretti, P.2013, 2013)

Según la Real Academia Española (RAE), citado por (Gutiérrez, I., 2017) la palabra inteligencia proviene del latín intelligentia, cuyas cinco primeras acepciones son:

1. f. Capacidad de entender o comprender.

2. f. Capacidad de resolver problemas.

3. f. Conocimiento, comprensión, acto de entender.

4. f. Sentido en que puede tomarse una proposición, un dicho o una expresión.

5. f. Habilidad, destreza y experiencia.

De esta manera, se puede entender que la definición de la palabra inteligencia se ha estado transformado y cambiando constantemente y en necesidad a la sociedad y a sus habilidades. Las primeras pruebas de inteligencia surgieron en 1904 con el psicólogo Alfred Binet en Francia y posteriormente se desarrollaron de una manera más amplia en Estados Unidos, en donde algunos psicólogos establecieron estándares para medir la inteligencia de una manera objetiva y reduciéndola a un puntaje llamado coeficiente intelectual. Quien estuviera por encima de la media era considerado genio y quien estuviera por debajo de la media era considerado débil mental. Bajo este modelo se trabajó durante muchos años. 
Del mismo modo, el autor Sternberg (1987) a su vez, considera la inteligencia como un autogobierno mental. Según este teórico, la inteligencia proporciona medios para gobernarnos a mismos, de manera que los pensamientos y acciones se organicen de manera coherente y responsable con respecto a las necesidades internas y del medio ambiente. Es entonces cuando surgen teorías con tendencia a explicar la inteligencia no sólo como un factor genético sino también dependiente de la cultura, la sociedad, el ambiente y otros factores que se explicará más adelante. (Sandoval, A., González, L., González, O., \& Lauretti, P.2013, 2013)

Continuando con esta temática, el autor dentro de las teorías que sustentan este concepto de varias inteligencias, se encuentra Spearman enunció en 1927 la teoría en la que afirma que la inteligencia está compuesta por varios factores, un factor general de carácter hereditario que interviene en todas las fases de la conducta humana y un factor especial que representa la habilidad de un sujeto frente a una tarea determinada. Ambos factores tendrían una localización concreta en el cerebro. (Gutiérrez, I., 2017)

Sobre el concepto de inteligencia se suele considerar a Francis Galton (1822-1911) citado en Fernández, Martín \& Domínguez (2006), como el primo de Darwin y como él interesado en los procesos de selección natural y la evolución, el primer estudioso de la inteligencia desde una perspectiva científica. El intento de definir la inteligencia viene de lejos; por ejemplo, Tomás de Aquino ya la definía como el conocimiento íntimo de las cosas, sin que hasta el momento se haya llegado a encontrar una definición que sea totalmente satisfactoria (Fernández, Martín y Domínguez, 2006).

Por su parte Wechsler (1958) citado en Fernández, Martín y Domínguez (2006) concibe la inteligencia como la suma o capacidad global de individuo para actuar de un modo provisto de finalidad, para pesar racionalmente y para tratar de enfrentarse de un modo eficaz a su medio ambiente. Un concepto íntimamente ligado al de inteligencia es el de aptitud. De forma general, se entiende por aptitud la capacidad individual para desempeñar una determinada tarea. Así, las pruebas que evalúan aptitudes buscan el grado de conocimiento, comprensión y habilidad sobre un tema o grupo de temas específicos.
De esta forma, se habla de aptitudes mecánicas, administrativas y de razonamiento, etcétera. Por su parte Gardner (2000) en cuanto a la definición de aprender de las personas, menciona que cada sujeto presenta características muy diferentes en la forma de aprender, es decir, posee un estilo de aprendizaje particular que le ayuda a interiorizar, de forma simple y permanente el conocimiento nuevo, haciéndolo significativo. De tal forma que cada uno experimenta y apropia los conocimientos de manera diferente.

Howard Gardner (1983) citado en (Romero Agudelo, L., \& Salinas Urbina, V., \& Mortera Gutiérrez, F., 2010), establece la Teoría de las inteligencias múltiples, este autor estableció originalmente siete estilos a saber la inteligencia verbal/lingüística; inteligencia lógica/matemática; inteligencia visual/espacial; inteligencia corporal/kinestética; inteligencia musical/rítmica; inteligencia interpersonal; e inteligencia intrapersonal, posteriormente se agrega la octava que es inteligencia naturalista. Gardner llegó a la conclusión de que las inteligencias trabajan en concierto unas con otras, por ello ante un déficit en un área del cerebro, el resto de las inteligencias podían cooperar entre ellas supliendo esa deficiencia. Por lo tanto, todos los seres humanos poseen como mínimo ocho inteligencias, en mayor o menor medida, si estas se estimulan de una manera sistemática el desarrollo es evidente (Gardner, 2012).

Ahora bien, Bonami et al., 2020 mencionan que "una idea sobre cómo definir la tecnología es el concepto de «technium»: un ecosistema autorreforzante de creación de artefactos, herramientas e ideas que abarcan las tecnologías. Según esta idea, la tecnología depende de innumerables avances anteriores".

El liderazgo considerado por algunos autores como un concepto mitológico Cohen \& March (1974), es una de las inquietudes más antiguas del mundo Bass (1990), y uno de los temas más elusivos, marginados y discutidos en las últimas décadas (Podolny, Khurana \& HillPopper (2005). Por ello el estudio del liderazgo está en la actualidad creciendo de forma acelerada y su investigación es cada vez más interdisciplinaria (Friedman, 2010). 
Se han establecido diferentes teorías acerca del liderazgo y se han debatido durante años si el liderazgo es una competencia que puede ser aprendida y desarrollada, o si es una propiedad propia del individuo (Chiavenato, 2009). Citados por León et al., 2017 p.188. Serrato \& Portalanza, 2014, citado por Pedraja et al., 2020, Nos dicen que a pesar de que el liderazgo ha sido un tópico de interés desde la antigüedad, este ha tomado fuerza desde el siglo XX, manteniéndose así hasta el día de hoy. En esta perspectiva y en el proceso de definición del concepto, el liderazgo ha sufrido cambios importantes, ya que en un principio las investigaciones de este eran realizadas desde una perspectiva individual, mientras que ahora es más bien considerada como una dinámica global, compleja, social y estratégica.(p.4)

Entre los retos que tiene el efectivo ejercicio del liderazgo, es que el entorno de trabajo se ha vuelto mucho más complejo en términos de la naturaleza y la dinámica de los desafíos que enfrentan los líderes (Mohanty et al., 2016). "El liderazgo es la capacidad de influir en un grupo para que se logren las metas" (Gómez, 2002). Para Davis \& Newstrom (2003), el liderazgo es el proceso de lograr influir sobre los demás con trabajo en equipo, con el propósito de que trabajen con entusiasmo en el logro de sus objetivos. Payeras (2004), lo explica como un grupo de formas de comportamiento que el líder usa como herramienta para influir sobre el actuar de los individuos y equipos. Esta influencia se desarrolla a través de una visión sustentada por los valores que la apoyan, generando en el individuo la incorporación a su propio comportamiento (Hellriegel \& Slocum, 2004). Entonces, el liderazgo organizacional es la capacidad de influir sobre los integrantes de los sistemas sociales para el logro de objetivos y metas.

\section{Metodología}

Una vez que se establece la interrogante general ¿Cómo se relacionan las TIC con las inteligencias múltiples y el desarrollo del liderazgo organizacional de los estudiantes de la UAdeC? se procede a indagar sobre la bibliografía que da sustento a la propuesta principal de este trabajo, se desarrolla el objetivo general y la hipótesis alternativa. Se elabora el instrumento de investigación y se efectúa la prueba piloto que permite hacer adecuaciones a los reactivos, la aplicación definitiva del cuestionario se ejecuta en una sesión.
Las TIC, las inteligencias múltiples y el desarrollo del liderazgo en los estudiantes entre 18 y 20 años serán predominantes para las formas de comportamiento que un líder usa como herramienta para influir sobre el actuar de los individuos.

Los datos obtenidos se organizan en una matriz de concentración y se les da tratamiento estadístico para explorar los resultados; se procesa la información en los análisis estadísticos, de frecuencias y porcentajes, correlación producto momento Pearson y con el integracional con el análisis factorial exploratorio; es una investigación cuantitativa, transversal de tipo descriptivo y correlacional.

Su hipótesis alternativa es:

$\mathrm{H}_{1}$ Existe una correlación directamente proporcional entre las TIC, las inteligencias múltiples y liderazgo organizacional.

\section{Resultados}

\section{Análisis Descriptivo}

\section{Frecuencias y Porcentajes}

De un total de 315 participantes estudiantiles, las edades de la muestra seleccionada, oscilan entre los 17 y 51 años de edad, de los cuales la mayor parte son mujeres (n:172, 55\%) y en menor porcentaje hombres (n:143, 45\%), por lo que conlleva que la carrera que más dio participación fue químicas (n:132,42\%), siendo así que predominan los estudiantes de sexto semestre (n:90, 28.57\%); en cuanto al uso de las TICS como herramienta educativa se observa que la mayoría de los estudiantes si hacen uso de ellas (n:274,86\%), a su vez, a la mayoría de los estudiantes no les cuesta dirigir un trabajo en equipo (n:209, 66.35\%); se muestra que la mayoría de los estudiantes conocen las inteligencias múltiples (n:223, 70.79\%).

\section{Análisis correlacional}

En este nivel se trabaja con correlación producto momento Pearson, con la varianza total del fenómeno de estudio a partir de las relaciones que existen. Dichas relaciones se fundamentan con una probabilidad de error de $\mathrm{p} \leq 0.01$. 
En los datos del análisis correlacional se destaca que cuando los estudiantes hacen uso de los TIC con la biblioteca digital para leer libros, documentos PDF y participación en foros en línea tienden a enriquecer su léxico; incrementando en ellos el comprender palabras poco usuales; a razonar antes de tomar alguna decisión y aumentar su habilidad para el análisis, además los beneficia a rescatar aspectos importantes de manera visual y fortalece las características de líder natural y el tener interés por los demás.

Cuando tienen una tarea definen qué TIC pueden emplear, utilizándolas de manera responsable, dicha herramienta les permite desarrollar experiencias de aprendizaje lo que les concede a ser más abiertos a opiniones y a tener características de líder natural.

En lo anterior se denota una correlación positiva con variables del uso de las TIC en relación con las inteligencias múltiples lingüística-verbal, lógico-matemático, espacial, interpersonal y con el liderazgo organizacional, se puede decir que las TIC de manera integrada con las inteligencias múltiples y con el liderazgo organizacional propicia ser un líder con inteligencia tecnológica.

\section{Análisis Integracional}

\section{Factorial exploratorio}

Con el interés de determinar la estructura que subyace en los temas estudiados, se procesa un análisis factorial exploratorio con rotación varimax. La prueba de adecuación muestral de Kaiser-Meyer-Olkin muestra un nivel de adecuación de 0.90, además la prueba de esferecidad de Barttlet rechaza la hipótesis nula por obtener un nivel crítico $($ Sig $)<0.05$; lo cual muestra que la matriz de correlaciones es adecuada para la factorización.

Para la retención de los factores se utiliza el criterio de sedimentación arrojando los siguientes factores:

\section{Factor 1. Coctel de inteligencias}

El factor 1 denominado coctel de inteligencias explica el $45.44 \%$ de la varianza total del fenómeno de estudio; está integrado por las variables lingüísticas, lógico-matemático y especial.
El análisis muestra que los universitarios tienen inclinación por el aprendizaje de nuevos idiomas, hacen uso de la ortografía al redactar un texto y siempre razonan antes de tomar una decisión, también tienen facilidad para resolver problemas matemáticos, utilizar los números eficazmente, hacer cálculos matemáticos mentalmente con bastante rapidez, tener habilidad para el análisis, les resulta fácil memorizar diferentes signos numéricos y siempre trabajar de manera metodológica, manifiestan habilidades para construir escenarios imaginarios, modificar superficialmente espacios, ser capaz de llevar a la realidad una idea, calcular el espacio físico, identificar detalles visualmente, pragmatizar los procesos a partir de la observación.

Se infiere que los universitarios que tienen interés por aprender nuevos idiomas, poseen la habilidad del análisis, del trabajo metodológico y de la observación.

\section{Factor 2. TIC e Inteligencias}

El factor dos denominado TIC e inteligencias, explica el $6.15 \%$ de la varianza total del fenómeno de estudio, integrado por las variables que conforman el uso de las TIC, de las inteligencias linguiísticas, lógico-matemático, espacial, musical, interpersonal e intrapersonal, y del eje de liderazgo organizacional

El análisis muestra lo relativo al uso de las TIC desde la biblioteca digital para leer libros en línea y documentos en pdf; lo que permite tener dominio de la inteligencia lingüística, dentro de la cual se caracteriza habilidad de asimilar lo que las personas expresan, en la inteligencia lógico-matemátise destaca la habilidad para el análisis además les resulta fácil memorizar diferentes signos numéricos.

Dentro de la inteligencia espacial se caracteriza por la habilidad de construir escenarios imaginarios, en cuanto a la inteligencia musical se caracteriza por recordar las melodías de las canciones, tararear inconsistentes tonadas musicales y responder favorablemente cuando alguien pone música, acciones que impactan en el ejercicio del liderazgo organizacional participativo en la toma de decisiones y abierto a opiniones. 
Se infiere que el conglomerado de estudiantes que realiza ciertas acciones TIC beneficia las inteligencias múltiples lingüísticas, lógico-matemático, espacial, musical, interpersonal e intrapersonal, lo que impacta en el ejercicio del liderazgo organizacional.

\section{Factor 3. Acto social}

El factor 3 denominado acto social explica el $4.32 \%$ de la varianza total del fenómeno de estudio; está integrado por las variables de liderazgo organizacional y TIC

En este factor se destaca que los sujetos manifiestan aptitudes de profesionalización que bien se pueden considerar loables, dado que a raíz de los resultados obtenidos se aprecia que son sujetos con una inclinación singular por tomar cursos y participar en foros virtuales, sin embargo, no solamente persiguen con estas actividades satisfacer sus propias metas o egos, sino que mediante los elementos adquiridos en los cursos y foros pretenden interactuar con la sociedad en forma significativa tanto en actividades dentro y fuera de la escuela, como en el área laboral.

Se infiere que los estudiantes que tienen la habilidad del uso de las TIC les interesa interactuar en forma significativa con los individuos en diferentes contextos.

\section{Factor 4. Líder Open-mind}

En el cuarto factor denominado líder open-mind con el $3.57 \%$ de la varianza total del fenómeno de estudio, el análisis muestra que los estudiantes consideran que un líder se caracteriza por saber escuchar, tener carisma y empatía, así como delegar responsabilidades. ser comunicador. que tenga alta motivación, iniciativa, muestre sentido de pertenencia, que elimine la competitividad, compartir conocimientos, que sea abierto a opiniones y mantenga el entusiasmo.

Se deduce que los estudiantes prefieren estar cerca de un líder en el que puedan tener más confianza y que sea motivador al momento de su desempeño personal.

\section{Factor 5. Tecnologías didácticas}

El quinto factor denominado tecnologías didácticas, explica el 3.33\% de la varianza total, integrado por la variable TIC'S dentro de la cual se caracteriza por utilizar el correo electrónico, uso de la biblioteca digital, escuchar música, ver películas, jugar videojuegos, visualizar las imágenes, mandar y recibir mensajes de texto, hacer videollamadas, leer libros en línea, leer documentos pdf, ver vídeos y editar de vídeos.

Se infiere que los participantes están dispuestos a utilizar las herramientas tecnológicas que se les brinda, para su beneficio como método de su aprendizaje.

\section{Conclusiones}

De acuerdo con los resultados obtenidos de los análisis estadísticos, en este apartado se presentan las conclusiones que muestran la perspectiva de Siendo Líder con Inteligencias Tecnológicas para dar respuesta a la interrogante principal, objetivo e hipótesis alternativa se menciona la siguiente:

Se concluye que la correlación positiva de variables del uso de las TIC en relación con las inteligencias múltiples lingüística-verbal, lógico-matemático, espacial, interpersonal y con el liderazgo organizacional propicia ser un líder con inteligencia tecnológica.

Se denota que los universitarios que tienen interés por aprender nuevos idiomas, poseen la habilidad del análisis, del trabajo metodológico y de la observación.

En conclusión el conglomerado de estudiantes que realiza ciertas acciones TIC beneficia las inteligencias múltiples lingüísticas, lógico-matemático, espacial, musical, interpersonal e intrapersonal, lo que impacta en el ejercicio del liderazgo organizacional.

Se muestra que los estudiantes que tienen la habilidad del uso de las TIC les interesa interactuar en forma significativa con los individuos en diferentes contextos.

Se deduce que los estudiantes prefieren estar cerca de un líder en el que puedan tener más confianza y que sea motivador al momento de su desempeño personal. 
Se concluye que los participantes están dispuestos a utilizar las herramientas tecnológicas que se les brinda, para su beneficio como método de su aprendizaje.

\section{Referencias}

Aguirre León, G., Serrano Orellana, B., \& Sotomayor Pereira, G. (2017). El liderazgo de los gerentes de las Pymes de Machala. Universidad y Sociedad [seriada en línea], 9 (1), pp. 187-195. http://rus.ucf.edu.cu/

Argudín, Y. (2006). Educación Basada en Competencias. 2a. Edición. México, Trillas. 109 pp.

Armstrong, T. (1999). Las Inteligencias Múltiples en el Aula. 1. Edición. Argentina. Manantial. 238 pp.

Bolivar, A. (2010). El liderazgo educativo y su papel en la mejora: una revisión actual de sus posibilidades y limitaciones.

Brunner J.J., (2000) "Educación escenarios de futuro. Nuevas tecnologías y sociedad de la transformación". Documento No. 16 OPREAL

Bonami,B.,Piazentini,L.,Dala,A.(2020)

Metodologías mixtas en plataformas digitales https://orcid.org/0000-0003-1995-6670)

Cebreiro C., Fernández C. (1997) "Las tecnologías de la comunicación en el espacio europeo para la educación superior"., Revista Científica de Comunicación y Educación, págs. 57-61.

Chiavenato, I. (2009). Gestión del Talento Humano. México: Mc Graw Hill.

Coll, C. (1991). Psicología y Currículum, 1 edición. México. Paidós.174 pp. (Cuadernos de Pedagogía).

Cruz J. Teorías del Aprendizaje y Tecnología de la enseñanza. México : Trillas, 2008.

Davis, K., \& Newstrom, J. (2003). Comportamiento humano en el trabajo. México: McGraw-Hill.

Fernández Zalazar, D. C. y Neri, C. (2011), Usos de las TICS en estudiantes universitarios y su relación con las estrategias de aprendizaje y estudio.
Gardner, H. (2012). Inteligencias múltiples. La teoría en la práctica. Barcelona: Paidos.

Garmen P., Rodríguez, C.,García, P.,Veledo, J., (2020) Inteligencias múltiples y videojuegos: Evaluación e intervención con software TOI Múltiple. http://orcid.org/0000-0001-7013-6518

Gómez, C. (2002). Liderazgo: conceptos, teorías y hallazgos relevantes. Cuadernos hispanoamericanos de psicología, 2(2), pp. 6177. Recuperado de http://www. uelbosque.edu.co/sites/default/files/publicacion es/revistas/cuadernos_hispanoamericanos_psico logia/volumen2_numero2/articulo_5.pdf

Gutiérrez, I. (septiembre-diciembre de 2017). La teoría de las inteligencias múltiples en personas con síndrome de Down. Cuando el talento se transforma en inteligencia. Obtenido de Investigación en Discapacidad: https://www.medigraphic.com/pdfs/invdis/ir2017/ir173d.pdf.

Hellriegel, D., \& Slocum, J. (2004). Comportamiento organizacional. México: Thomson.

Jiménez E. (2016). La dirección de los centros educativos, entre el ideal y la realidad.

Mohanty, J., Ara Begum, J., y Kar, B., (2016) Nuances of Leadership Effectiveness: Challenges of Context and Demographics, https://doi.org/10.21863/ijl/2016.4.1.013,

International Journal on Leadership, 4(1), 15-25

Murillo, F. J. (2017). Avances en liderazgo y mejora de la educación. Actas del I Congreso Rojas, A. y Gaspar, F. (2006). Bases del liderazgo en educación.

Pedraja-Rejas, Ítalo A. Marchioni-Choque, Constanza J. Espinoza-Marchant y Camila P. Muñoz-Fritis., (2020) Liderazgo y cultura organizacional como factores que influyen en la calidad de la educación superior: el análisis conceptual. El formulario. Univ. [en línea]. 2020, vol.13, n.5, pp.3-14. ISSN 0718-5006. http://dx.doi.org/10.4067/S071850062020000500003.. 
Sánchez-Otero, M., García-Guiliany, J., Steffens-Sanabria, E., \& Hernández- Palma, H. (2019). Pedagogical Strategies in Teaching and Learning Processes in Higher Education including Information and Communication Technologies. Informacion Tecnologica, 30(3), 277-286. https://doi.org/10.4067/S071807642019000300277

Payeras, 2. (2004). Coaching y Liderazgo: Para directivos interesados en incrementar sus resultados. España: Díaz de Santos.

Sandoval, A., González, L., González, O., \& Lauretti, P.2013. (2013). Inteligencia naturalista y existencial: una contribución al desarrollo emocional y al bienestar. Obtenido de Multiciencias:

http://www.redalyc.org/pdf/904/90429040009.p df

Romero Agudelo, L., \& Salinas Urbina, V., \& Mortera Gutiérrez, F. (2010). Estilos de aprendizaje basados en el modelo de Kolb en la educación virtual. Obtenido de Apertura, 2 (1) : http://www.redalyc.org/articulo.oa?id=6882084 1007

Vasović, N., y D. Milašinović, (2014) Modern Information and Communication Technologies in Higher Education, In $7^{\text {th }}$ International conference Higher Education in Function of Sustainable Development, Užice. 